\title{
High ALDH1 expression correlates with better prognosis in tumorigenic malignant melanoma
}

\author{
Laura A Taylor ${ }^{1,6}$, Ronnie M Abraham ${ }^{2,6}$, Emin Tahirovic ${ }^{3}$, Patricia van Belle ${ }^{1}$, Bin $\mathrm{Li}^{4}$, \\ Linfang Huang ${ }^{5}$, David E Elder ${ }^{1}$, Phyllis Gimotty ${ }^{3}$ and Xiaowei $\mathrm{Xu}^{1}$
}

${ }^{1}$ Department of Pathology and Laboratory Medicine, Hospital of the University of Pennsylvania, Philadelphia, PA, USA; ${ }^{2}$ Departments of Pathology and Dermatology, University of Illinois College of Medicine at Peoria, Peoria, IL; ${ }^{3}$ Department of Biostatistics and Epidemiology, Hospital of the University of Pennsylvania, Philadelphia, PA, USA; ${ }^{4}$ Department of Dermatology, Yueyang Hospital, Shanghai, China and ${ }^{5}$ Peking Union Medical College, Chinese Academy of Medical Sciences, Beijing, China

\begin{abstract}
Aldehyde dehydrogenase 1 (ALDH1) has been proposed as biomarker of stem cells for certain human cancers. ALDH1 expression has been correlated with poor patient outcomes in a variety of malignancies but better patient outcomes in others, and its prognostic significance in malignant melanoma is unclear. Thus, 68 melanoma patients with comprehensive clinical and pathologic follow-up data were used to construct a tissue microarray. A modified histological score (H-score) with a maximum score of $\mathbf{3 0 0}$ was used to quantify immunohistochemical staining for ALDH1. Survival time was defined as the time between diagnosis and melanoma-specific death. Using univariate logistic regression, a low ( $<80 \mathrm{H}$-score) ALDH1 score showed 3.7-fold increase in risk for melanoma-specific death within 10 years when compared with high ( $>80)$ ALDH1 levels $(P=0.017)$. Odds of MSD were lower by a factor of $\sim 0.9$ for each 10-point increase in H-Score. Median survival time was 44.1 months and 180.9 months for patients with low and high ALDH1 expression, respectively. Using multivariate analysis, ALDH1 $\mathrm{H}$-score was found to be an independent prognostic factor. These findings suggest that ALDH1 expression in malignant melanoma has a favorable effect on patient survival. Further study is needed elucidate the function of this enzymatic protein in melanoma progression.
\end{abstract}

Modern Pathology (2017) 30, 634-639; doi:10.1038/modpathol.2016.226; published online 20 January 2017

Reported incidence of malignant melanoma has been increasing in the United States since the $1930 \mathrm{s.} .^{1,2}$ Although it accounts for $<1 \%$ of skin cancer it is responsible for $\sim 75 \%$ of skin cancer-related deaths. ${ }^{3}$ Owing to its aggressive nature and its ability to acquire resistance to many standard therapies there is significant interest in understanding the targetable molecular pathways involved in its tumorigenesis in hopes of discovering new and effective treatment alternatives. ${ }^{4}$ It has been hypothesized that tumor initiation and therapy resistance may be associated with cells exhibiting so-called cancer stem cell ${ }^{5}$

Correspondence: Professor P Gimotty, PhD, Department of Biostatistics and Epidemiology, University of Pennsylvania Perelman School of Medicine, 423 Guardian Drive, 631 Blockley Hall, Philadelphia, PA 19104-6021, USA and Professor X Xu, MD, PhD, Department of Pathology and Laboratory Medicine, University of Pennsylvania, 3400 Spruce St., 6 Founders Building, Philadelphia, PA 19104, USA.

E-mail: pgimotty@mail.med.upenn.edu and xug@mail.med.upenn.edu ${ }^{6}$ These authors contributed equally to this work.

Received 16 June 2016; revised 21 November 2016; accepted 23 November 2016; published online 20 January 2017 properties. Levels of activity of the hypothesized cancer stem cell marker aldehyde dehydrogenase $(\mathrm{ALDH})$, and in particular its isoform 1, have been shown to be consistent with cancer stem cell activity in a variety of cancers. ${ }^{6-14}$ Unfortunately studies of melanoma have yielded conflicting results. ${ }^{15-19}$

The level of activity of ALDH1, which belongs to a family of ubiquitous detoxifying enzymes that function to convert aldehydes to their respective carboxylic acids, ${ }^{20,21}$ is associated with poor clinical outcomes in breast cancer ${ }^{6}$ and leukemia, ${ }^{13}$ but correlates with better clinical outcomes in ovarian cancer ${ }^{22}$ and non-small-cell lung cancer. ${ }^{23}$ Recently, a study testing chemotherapeutics in melanoma xenograft mice models has shown promising results for ALDH-targeted therapies demonstrating the need for increased understanding of this pathway and its role in disease progression. ${ }^{19}$ However, to date no investigations have directly assessed the prognostic significance of ALDH1 in melanoma. In this paper, we investigate the relationship between clinical outcomes and ALDH1 activity in melanoma. 


\section{Materials and methods}

\section{Study Patients and Design}

The 68 patients eligible for this retrospective study of a prospective cohort had vertical growth phase tumorigenic primary melanomas, no regional nodal procedure or apparent metastases at the time of definitive treatment between 1972 and 1991 at the University of Pennsylvania's Pigmented Lesion Clinic, and at least 10 years of follow-up. They had at least 10 years of protocol-driven, prospective follow-up and had paraffin blocks available for tissue microarray. The protocol was approved by the Institutional Review Board of the University of Pennsylvania.

\section{Tissue Microarray Construction and Immunohistochemical Staining}

Tissues from 68 patients were included in the tissue microarray. These patients had vertical growth phase primary melanomas and no regional nodal procedure or apparent metastases at the time of definitive treatment between 1972 and 1991 at the University of Pennsylvania's Pigmented Lesion Clinic. The tissue microarray was composed of $2 \mathrm{~mm}$ cores. When we designed the tissue microarray, we considered the possibility of heterogeneity within melanomas. Sixty-three percent of cases had three cores from different areas of primary melanomas and thirty-four percent of the cases had four cores. Two cases had one or two cores owing to smaller primary tumor volumes. Immunohistochemical staining was performed for ALDH1 antibody (BD biosciences) using a 1:800 dilution with citrate retrieval. ALDH1 expression was analyzed by two independent pathologists (RMA, XX) using a modified histological score (H-score) based both on the percentage of positively stained cells and on the intensity of staining, with a maximum score of 300 (see Figure 1). For statistical analysis, the scores for each core reported by the two readers were averaged. The tissue microarray was constructed at the Penn tissue microarray facility in the Department of Pathology and Laboratory Medicine.

\section{Pathology Definitions}

All original hematoxylin and eosin-stained slides were read at the time of definitive treatment by pathologists associated with the Pigmented Lesion Clinic of the University of Pennsylvania (chiefly David E Elder and Wallace H Clark, Jr). Attributes recorded at the time of diagnosis include thickness and anatomical level; dermal mitotic rate, expressed in terms of mitoses per square millimeter; vertical growth phase tumorinfiltrating lymphocytes, classified as either brisk, nonbrisk, or absent; regression, if present in the radial growth phase that often accompanies the vertical growth phase; microscopic satellites; ulceration; and vascular (blood or lymphatic) invasion. For statistical analysis, some characteristics were converted to categorical variables: thickness $(1.01-2.00 \mathrm{~mm}, 2.01-$ $4.00 \mathrm{~mm}$, or $4.01 \mathrm{~mm}$ and more); mitotic rate ( $<1$ and $\geq 1$ mitoses $/ \mathrm{mm}^{2}$ ) and Clark's level (III, IV/V).

\section{Clinical Definitions}

Clinical characteristics available for all patients included age, sex, and the anatomic site of the primary lesion. For statistical analysis, binary variables were created for age at diagnosis ( $\geq 60$ years, $<60$ years) and anatomic site (extremity, axial). Survival time was defined as the time between definitive treatment and melanoma-specific death. The 10-year melanoma-specific death rate was defined as the proportion of patients who died of melanoma within 10 years of definitive treatment.

\section{Statistical Methods}

Univariate logistic regression analyses were used to estimate unadjusted odds ratios for 10-year
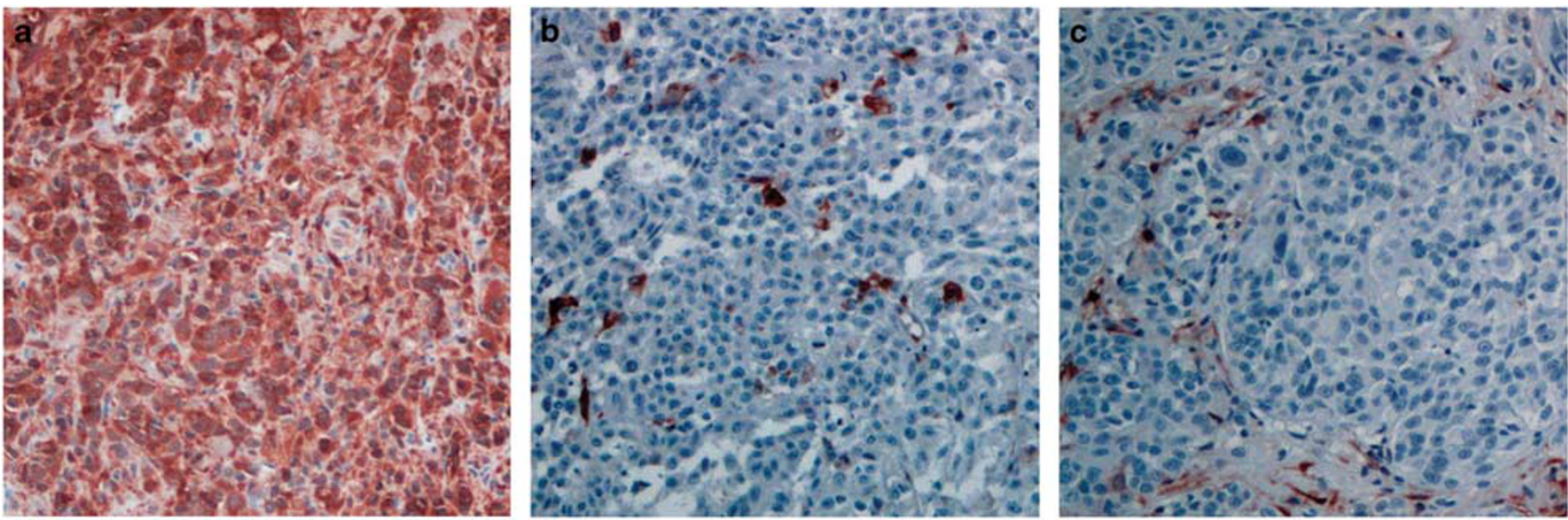

Figure 1 Variations in ALDH1 Immunohistochemical staining of Melanoma. Section A shows strong diffuse staining in the melanoma cells with ALDH1. Section B shows scattered positivity, whereas section C shows no staining within the lesional cells. 
melanoma-specific death and H-score as well as each of the other 10 available factors. The three factors that were significantly associated with 10-year melanomaspecific death in univariate models were included in a multivariate model. One factor that that did not make a significant contribution to the multivariate model was removed based on a likelihood ratio test to obtain a parsimonious, reduced multivariate model. Based on the number of events in this sample, 2-3 variables in the regression model are recommended to avoid overfitting (See Harrell, 2015, pp. 72-74). ${ }^{24}$ X-tile software ${ }^{25}$ was used to compute optimal
H-score subgroup thresholds (two thresholds, and thus 'low', 'middle', and 'high' groups). These thresholds were determined by computing the two KaplanMeier survival curves defined by all possible $\mathrm{H}$-score cut-points, and the associated log-rank statistics to identify the cut-point where the two curves were most different based on the log-rank statistics. Exact $\chi^{2}$-tests (SAS Proc FREQ) were used to compare distributions for pathological and clinical factors among the H-Score subgroups. All statistical analyses were performed using SAS/STAT software, Version 9.4 of the SAS System for PCs.

Table 1 Patient and tumor characteristics, overall and by H-score subgroup

\begin{tabular}{|c|c|c|c|c|c|}
\hline & \multirow{2}{*}{$\begin{array}{c}\text { All patients } \\
\mathrm{n}=68 \text { Percent }\end{array}$} & \multicolumn{4}{|c|}{$H$-score subgroups } \\
\hline & & $\begin{array}{c}\text { Low }(0-2) \mathrm{n}=10 \\
\text { Percent }\end{array}$ & $\begin{array}{c}\text { Intermediate }(3-120) \mathrm{n}=43 \\
\text { Percent }\end{array}$ & $\begin{array}{l}\text { High }(121-300) \mathrm{n}=15 \\
\text { Percent }\end{array}$ & P-value ${ }^{\mathrm{a}}$ \\
\hline Age at diagnosis & & & & & 0.430 \\
\hline$<60$ years & 55.9 & 40 & 55.8 & 66.7 & \\
\hline$\geq 60$ years & 44.1 & 60 & 44.2 & 33.3 & \\
\hline Sex & & & & & 0.564 \\
\hline Male & 67.6 & 80 & 62.8 & 73.3 & \\
\hline Female & 32.4 & 20 & 37.2 & 26.7 & \\
\hline Anatomic site & & & & & 0.863 \\
\hline Axial & 72.1 & 80 & 69.8 & 73.3 & \\
\hline Extremity & 27.9 & 20 & 30.2 & 26.7 & \\
\hline Thickness & & & & & 0.770 \\
\hline $1.01-2.00 \mathrm{~mm}$ & 14.7 & 20 & 14.0 & 13.3 & \\
\hline $2.01-3.00 \mathrm{~mm}$ & 27.9 & 10 & 30.2 & 33.3 & \\
\hline $3.01-9.00 \mathrm{~mm}$ & 57.4 & 70 & 55.8 & 53.3 & \\
\hline Regression & & & & & $0.055^{\mathrm{c}}$ \\
\hline Present & 44.1 & 50 & 44.1 & 84.6 & \\
\hline Absent & 36.8 & 50 & 55.9 & 15.4 & \\
\hline Unknown & 19.1 & - & - & - & \\
\hline Mitotic rate ${ }^{b}$ & & & & & $0.645^{\mathrm{c}}$ \\
\hline$<1.0$ & 8.8 & 10 & 4.8 & 13.3 & \\
\hline $1.0-40.0$ & 89.7 & 90 & 95.2 & 86.7 & \\
\hline Unknown & 1.5 & - & - & - & \\
\hline TIL & & & & & 0.912 \\
\hline Present & 77.9 & 80 & 79.1 & 73.3 & \\
\hline Absent & 22.1 & 20 & 20.9 & 26.7 & \\
\hline Ulceration & & & & & $0.349^{\mathrm{C}}$ \\
\hline Present & 45.6 & 70 & 45.0 & 42.9 & \\
\hline Absent & 48.5 & 30 & 55.0 & 57.1 & \\
\hline Unknown & 5.9 & - & - & - & \\
\hline Clark’s level & & & & & 0.016 \\
\hline III & 44.1 & 20 & 39.5 & 73.3 & \\
\hline IV \& V & 55.9 & 80 & 60.5 & 26.6 & \\
\hline Satellites & & & & & 0.331 \\
\hline Present & 27.9 & 40 & 30.2 & 13.3 & \\
\hline Absent & 72.1 & 60 & 69.8 & 86.7 & \\
\hline
\end{tabular}

Abbreviation: TIL, tumor-infiltrating lymphocytes.

${ }^{\mathrm{a}}$ Exact $\chi^{2}$-test for equality of proportions across the three H-score subgroups.

${ }^{\mathrm{b}}$ Mitoses $/ \mathrm{mm}^{2}$.

${ }^{\mathrm{C} U n k n o w n}$ values excluded from the analysis. 


\section{Results}

The ALDH1 immunohistochemical staining pattern was primarily cytoplasmic. We noted heterogeneity of ALDH1 staining in individual tumor cells (Figure 1). However, the variability within a tumor is relatively uniform which is reflected by similar $\mathrm{H}$-scores of different cores from the same case. We stained some whole sections of melanomas with ALDH1 and confirmed that the variability in staining was relatively even within a melanoma. There was no specific distribution of positive cells in relationship to tumor necrosis or perivascular localization.

Table 1 presents the clinical and histopathologic characteristics for the 68 study patients. The median age at diagnosis was $\sim 59$ years (range, $21-82$ years). The median thickness was $3.25 \mathrm{~mm}$ (range, 1.04$8.86 \mathrm{~mm})$. The majority of patients were Clark's level IV/V (56\%), 46\% exhibited ulceration, and satellites were present in $72 \%$ of cases. Median mitotic rate was $6.3 \mathrm{mitoses} / \mathrm{mm}^{2}$ (range, 0-38.9 mitoses $/ \mathrm{mm}^{2}$ ). The mean H-Score was computed for each patient using either four cores (34\%), three cores $(63 \%)$ or one or two cores $(2.7 \%)$. The median H-score was 27 (range, 0-241). Within 10 years of definitive excision of their primary lesions, 42 patients died of causes related to their disease, resulting in a 10-year melanoma-specific death rate of $62 \%$.

Three factors were significantly associated with 10-year melanoma-specific death in the univariate logistic regression analyses (Table 2, Univariate Models). ALDH1 H-score was inversely associated with the likelihood of 10-year melanoma-specific death (odds ratio $=0.91, P=0.020$ ), indicating that for each 10-point increase in ALDH1 H-score, the odds of 10-year melanoma-specific death decreases by a factor of 0.91. Both microscopic satellites (odds ratio $=4.72, P=0.025$ ) and Clark level IV $/ \mathrm{V}$ (odds ratio $=3.20, P=0.025$ ) were associated with a higher odds of 10-year melanoma-specific death compared with patients without microscopic satellites and for patients with Clark level III melanomas, respectively.

The multivariate logistic regression model included the three factors: ALDH1 H-score, the presence of satellites, and Clark's level, (see Table 2 Multivariate

Table 2 Logistic regression analysis for 10-year melanoma-specific death $(n=68)$

\begin{tabular}{|c|c|c|c|c|c|c|}
\hline & \multicolumn{2}{|c|}{ Univariate models } & \multicolumn{2}{|c|}{ Multivariate model ${ }^{\mathrm{a}}$} & \multicolumn{2}{|c|}{ Reduced model } \\
\hline & Unadjusted OR & $\mathrm{P}$-value & Adjusted OR & $\mathrm{P}$-value & Adjusted OR & $\mathrm{P}$-value \\
\hline Modified H-score ${ }^{b}$ & 0.91 & 0.020 & 0.93 & 0.089 & 0.92 & 0.034 \\
\hline \multicolumn{7}{|l|}{ Satellites } \\
\hline Present & 4.72 & 0.025 & 3.78 & 0.070 & 4.35 & 0.040 \\
\hline Absent & 1.00 & - & 1.00 & - & 1.00 & - \\
\hline \multicolumn{7}{|l|}{ Clark's level } \\
\hline III & 1.00 & 0.025 & 1.00 & 0.283 & - & - \\
\hline IV \& V & 3.2 & - & 1.86 & - & - & - \\
\hline Thickness (mm) & 1.34 & 0.060 & - & - & - & - \\
\hline Mitotic rate ${ }^{\mathrm{C}}$ & 1.05 & $0.273^{\mathrm{d}}$ & - & - & - & - \\
\hline Age at diagnosis (years) & 1.01 & 0.425 & - & - & - & - \\
\hline \multicolumn{7}{|l|}{ Sex } \\
\hline Male & 0.89 & 0.826 & - & - & - & - \\
\hline Female & 1.00 & - & - & - & - & - \\
\hline \multicolumn{7}{|l|}{ Anatomic site } \\
\hline Axial & 2.29 & 0.133 & - & - & - & - \\
\hline Extremity & 1.00 & - & - & - & - & - \\
\hline \multicolumn{7}{|l|}{ Regression } \\
\hline Present & 0.76 & $0.540^{\mathrm{d}}$ & - & - & - & - \\
\hline Absent & 1.00 & - & - & - & - & - \\
\hline \multicolumn{7}{|l|}{$T I L$} \\
\hline Present & 0.76 & 0.659 & - & - & - & - \\
\hline Absent & 1.00 & - & - & - & - & - \\
\hline \multicolumn{7}{|l|}{ Ulceration } \\
\hline Present & 1.34 & $0.570^{\mathrm{d}}$ & - & - & - & - \\
\hline Absent/unknown & 1.00 & - & - & - & - & - \\
\hline
\end{tabular}

Abbreviation: OR, odds ratio.

${ }^{\mathrm{a}}$ Multivariate model included the three variables significant in the univariate analyses.

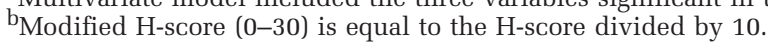

${ }^{\mathrm{C}}$ Mitoses per high powered field.

${ }^{d}$ Unknown data excluded from the analysis. 


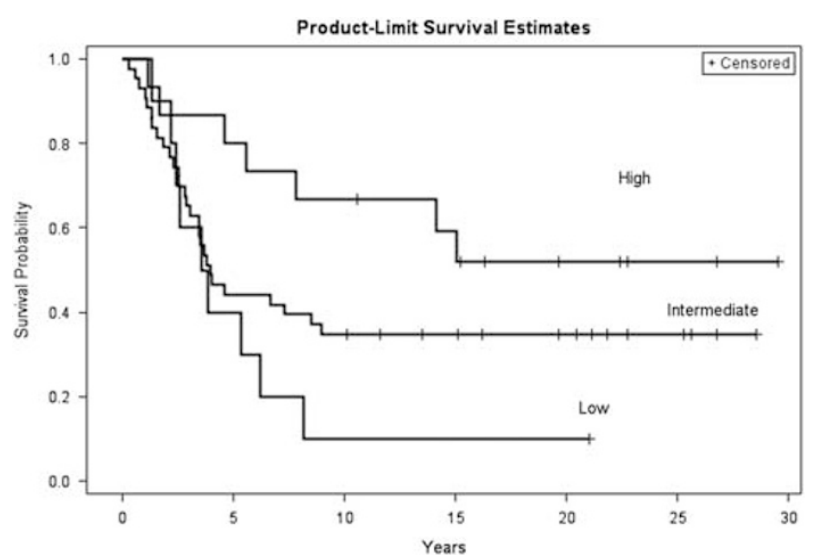

Figure 2 Survival curves for time to melanoma-specific death by subgroups defined by optimal cut-points for ALDH1 H-score. Low ALDH1 included those with an $\mathrm{H}$-score between 0 and 2, intermediate ALDH1 included those with an $\mathrm{H}$-score between 3 and 120, and high ALDH1 included those with an H-score between 121 and 300

model). The reduced multivariate model included ALDH1 H-score (odds ratio $=0.92, P=0.034$ ) and microscopic satellites (odds ratio $=4.35, P=0.040$ ) as independent prognostic factors for 10-year melanoma-specific death (Table 2, reduced model). The interpretations and magnitudes of these coefficients are similar to those from the univariate models.

To further investigate the relationship between survival and ALDH1 expression, two optimal cutpoints for $\mathrm{H}$-score were determined to examine characteristics of those with low, intermediate and high risk of 10-year melanoma-specific death. The two optimal cut-points were estimated to be 2.83 and 120 and three subgroups were defined: low ALDH1 (H-score between 0 and 2), intermediate ALDH1 (H-score between 3 and 119), and high ALDH1 (H-score between 120 and 300) (Figure 2). The 'high' group had significantly more lesions having Clark's level III $(73 \%$ in the high group versus $20 \%$ and $40 \%$ in low and medium groups, $P=0.016$ ) and had marginally significantly more lesions with radial growth phase regression $(73 \%$ in the high group versus $40 \%$ and $35 \%$ in low and medium groups, $P=0.055$ ).

\section{Discussion}

In this study of 68 patients with tumorigenic malignant melanoma, comprehensive staging and long-term clinical follow-up, high levels of ALDH1 activity as interpreted by immunohistochemical staining was associated with better patient outcomes. Odds of 10-year melanoma-specific death was lower by a factor of $\sim 0.9$ for each 10-point increase in $\mathrm{H}$-Score. Using multivariate analysis, ALDH1 $\mathrm{H}$-score was also found to be an independent prognostic marker $(P$-value $=0.034)$. Overall, the data support that higher ALDH1 activity in this cohort correlated with better prognosis.

As noted above, studies have linked ALDH1 levels with both positive and negative prognostic significance depending on the type of cancer studied. Our finding of higher levels of ALDH1's association with better prognosis in melanoma mirrors similar findings in non-small cell lung cancer ${ }^{23,26}$ but contrasts with data on breast cancer ${ }^{6,11}$ and leukemia, ${ }^{13}$ where higher ALDH1 levels correlated with poor prognosis. These apparent discrepancies between ALDH1 expression in different tumors and clinical outcomes could reflect the notion that ALDH1, a ubiquitous metabolic enzyme, may serve different functions in each organ system, and thus may have a specific role in each organ's tumor biology. Its positive correlation with prognosis suggests that ALDH1 expression is unlikely to serve as a cancer stem cell marker for melanoma in the way it may in breast cancer, where ALDH1 expression is believed to correlate with cancer stem cell status. Moreover, it may be a marker of differentiation rather than undifferentiation.

ALDH1 is important for tumor cell proliferation, survival, and resistance to chemotherapeutic agents. Small molecules that inhibit ALDH1 functions have been developed, such as diethylaminobenzaldehyde and disulfiram. Therefore, targeted therapy to ALDH1 may be tested in preclinical models and future clinical trials. However, as noted above, the effects of ALDH1 expression on tumor growth and metastasis appear to be tumor-type specific. To better target ALDH1 for therapy, additional studies are needed to understand the mechanisms underlying functional role of ALDH1 in different tumor types.

\section{Acknowledgments}

This work was supported by grants P50-CA-093372 (XX, PG), P30-CA016520 (PG), P50-CA174523 (XX, PG), and PO1CA114046 (XX) from the National Institutes of Health.

\section{Disclosure/conflict of interest}

The authors declare no conflict of interest.

\section{References}

1 Rigel DS. Epidemiology of melanoma. Semin Cutan Med Surg 2010;29:204-209.

2 Siegel R, Naishadham D, Jemal A. Cancer statistics. 2013CA Cancer J Clin 2013;63:11-30.

3 American Cancer Society. Cancer Facts and Figures 2016. http://www.cancer.org/acs/groups/content/@research/ documents/document/acspc-047079.pdf. 2016.

4 Agarwala SS. Current systemic therapy for metastatic melanoma. Expert Rev Anticancer Ther 2009;9:587-595.

5 Reya T, Morrison SJ, Clarke MF, Weissman IL. Stem cells, cancer, and cancer stem cells. Nature 2001;414: 105-111. 
6 Ginestier C, Hur MH, Charafe-Jauffret E, et al. ALDH1 is a marker of normal and malignant human mammary stem cells and a predictor of poor clinical outcome. Cell Stem Cell 2007;1:555-567.

7 Deng S, Yang X, Lassus $\mathrm{H}$, et al. Distinct expression levels and patterns of stem cell marker, aldehyde dehydrogenase isoform 1 (ALDH1), in human epithelial cancers. PLoS One 2010;5:e10277.

8 Gasparetto M, Sekulovic S, Zakaryan A, et al. Varying levels of aldehyde dehydrogenase activity in adult murine marrow hematopoietic stem cells are associated with engraftment and cell cycle status. Exp Hematol 2016;40:857-866.

9 Luo W-R, Gao F, Li S-Y, Yao K-T. Tumour budding and the expression of cancer stem cell marker aldehyde dehydrogenase 1 in nasopharyngeal carcinoma. Histopathology 2012;61:1072-1081.

10 Ma S, Chan KW, TK-W Lee, et al. Aldehyde dehydrogenase discriminates the CD133 liver cancer stem cell populations. Mol Cancer Res 2008;6:1146-1153.

11 Morimoto K, Kim SJ, Tanei T, et al. Stem cell marker aldehyde dehydrogenase 1-positive breast cancers are characterized by negative estrogen receptor, positive human epidermal growth factor receptor type 2, and high Ki67 expression. Cancer Sci 2009;100:1062-1068.

12 Ozbek E, Calik G, Otunctemur A, et al. Stem cell markers aldehyde dehydrogenase type 1 and nestin expressions in renal cell cancer. Arch Ital Urol Androl 2012;84:7-11.

13 Ran D, Schubert M, Pietsch L, et al. Aldehyde dehydrogenase activity among primary leukemia cells is associated with stem cell features and correlates with adverse clinical outcomes. Exp Hematol 2016;37:1423-1434.

14 Suzuki E, Chiba T, Zen Y, et al. Aldehyde dehydrogenase 1 is associated with recurrence-free survival but not stem cell-like properties in hepatocellular carcinoma. Hepatol Res 2012;42:1100-1111.

15 Prasmickaite L, Engesaeter BO, Skrbo N, et al. Aldehyde dehydrogenase (ALDH) activity does not select for cells with enhanced aggressive properties in malignant melanoma. PLoS One 2010;5:e10731.

16 Luo Y, Dallaglio K, Chen Y, et al. ALDH1A isozymes are markers of human melanoma stem cells and potential therapeutic targets. Stem Cells 2012;30:2100-2113.

17 Luo Y, Nguyen N, Fujita M. Isolation of human melanoma stem cells using ALDH as a marker. Curr Protoc Stem Cell Biol 2012;2013-2014.

18 Boonyaratanakornkit JB, Yue L, Strachan LR, et al. Selection of tumorigenic melanoma cells using ALDH. J Invest Dermatol 2010;130:2799-2808.

19 Yue L, Huang Z-M, Fong S, et al. Targeting ALDH1 to decrease tumorigenicity, growth and metastasis of human melanoma. Melanoma Res 2015;25:138-148.

20 Lindahl R. Aldehyde dehydrogenases and their role in carcinogenesis. Crit Rev Biochem Mol Biol 1992;27: 283-335.

21 Jackson B, Brocker C, Thompson DC, et al. Update on the aldehyde dehydrogenase gene (ALDH) superfamily. Hum Genomics 2011;5:283-303.

22 Chang B, Liu G, Xue F, et al. ALDH1 expression correlates with favorable prognosis in ovarian cancers. Mod Pathol 2009;22:817-823.

23 Dimou A, Neumeister V, Agarwal S, Anagnostou V, Syrigos K, Rimm DL. Measurement of aldehyde dehydrogenase 1 expression defines a group with better prognosis in patients with non-small cell lung cancer. Am J Pathol 2012;181:1436-1442.

24 Harrell F. Regression Modeling Strategies: With Applications to Linear Models, Logistic and Ordinal Regression, and Survival Analysis. Cham, Switzerland: Springer International Publishing, 2015; https://books. google.com/books?id = 94RgCgAAQBAJ.

25 Camp RL. X-Tile: a new bio-informatics tool for biomarker assessment and outcome-based Cut-point optimization. Clin Cancer Res 2004;10:7252-7259.

26 Jiang F, Qiu Q, Khanna A, et al. Aldehyde dehydrogenase 1 is a tumor stem cell-associated marker in lung cancer. Mol Cancer Res 2009;7:330-338. 